the bodies in it, but such theories can obviously be nothing more than rational pictures painted in the pigments available at the time. As they must change with the expansion of theoretical and observational knowledge, they can never be more than temporary schemes which explain what is known when they are expressed, and suggest a possible past and future history from present appearances. Mathematics and philosophy are, however, not confined to known laws of Nature or to observable phenomena: though these may be used in eonstructing mechanical systems of the universe, they are not essential factors in mathematical conceptions, any more than they are in poetic flights of fancy. Ideas as to the origin and construction of the universe, based on logical mathematical principles, can only be refuted, therefore, by positive evidence of their untruth, and not by the apparent lack of contact with what is known when they are put forward.

Our senses determine the range of objective phenomena, but creative thought has no such limitations. It is the source of the greatest human achievements, whether expressed in music and poetry or in scientific discovery and invention. Its exercise is determined not by what is known but by what is unknown; and whether a pursuit is worth while must be measured by originality of intention and result rather than by direct intellectual or practical service. Here, then, is the common standard by which all scientific inquiries, and all expressions of human feelings, may be judged. It makes no distinction between pure and applied science, so long as the object is increase of knowledge and the endeavour is the discovery of truth.

When this is borne in mind, the pursuit of knowledge for its own sake becomes just as estimable an occupation as that in which the purpose is use or action. It is generally acknowledged that inquiries undertaken to solve purely scientific problems, and without thought of their proximate or ultimate usefulness, have been the starting points of most of the great achievements of modern science; but such problems need not be excluded in planning scientific work for the benefit of the community. Science has transformed so many aspects of modern civilization that structures of society designed in earlier times have been shaken to their foundations by it. Its sources and resources, if they are wisely used, give almost unlimited powers to construct a world in which life can be made worth living to all peoples of the earth.

Systems of planning with these objects in view have to provide not only for the full use of existing knowledge but also for efficient means of extending it. Most scientific inquiries are best advanced when groups of workers concentrate attention upon them, whether intellectual interest or industrial application is the motive. It is, however, as impossible as it is undesirable to attempt to limit creative thought to a particular pattern, or to apply the criterion of usefulness to its exercise. This is as true of science as it is of other activities in which hand and brain combine to express themselves in new products. Men of science, like musical composers and other artists, may follow their occupations as a means of living, but their most original achievements are those which depend for their expression upon inborn light rather than external influences. In every walk in life, both interest and pleasure are required for contented effort, but they are not always to be hired in the market place. They are at their best when they are exercised in perfect freedom, whether in craftsmanship or in the expression of human consciousness.

\title{
STRUCTURE OF LIQUID METALS
}

\author{
By Dr. A. LATIN \\ Research Department, Messrs. Thomas Bolton and Sons, Ltd.
}

$\mathrm{T}$ $\mathrm{HE}$ view that the arrangement of atoms or molecules in a liquid is not entirely random may now be considered well established. In other words, the liquid possesses certain characteristics to which the term 'structure' can be properly applied. This does not mean that a liquid possesses anything so definite as a 'lattice structure', and any reference to a 'liquid lattice' may be very misleading, if not, indeed, devoid of meaning. It would perhaps be allowable to refer to the 'ghost of a lattice. Before going on to consider liquid metals themselves, it will be as well to deal first with the general question of the structure of liquids.

There are perhaps two main types of conception of liquid structure that have proved of value. These may be termed (1) group and (2) statistical conceptions. In some ways, indeed, these con. 
ceptions have tended to merge into one another and perhaps their chief distinction is in the difference of mental picture that they give. Thus one view or the other may prove of the more value according to the particular case under consideration.

Theories of the first type postulate the existence in the liquid of minute groups of atoms or molecules possessing a more or less high degree of order, particularly at temperatures near the melting point. Such theories have been put forward, for example, to account for the increase in supercooling on freezing with the degree of original superheat. This implies a higher degree of order (or a greater number of 'seed crystals') in a freshly melted liquid than in one which has been superheated. There is considerable evidence, however, favouring the alternative view that the effect is due to the presence of impurities which, in one way or another, furnish nuclei for crystallization. Even in the case of the so-called 'liquid crystals', there appears to be no evidence of any very definite regularity of arrangement of the molecules, other than a common orientation. Perhaps the most elegant development of the group type of conception is Stewart's theory of 'cybotaxis'. In this the groups are considered to be of a transitory nature, continually forming and disappearing. The mental picture of the liquid is, so to speak, 'spotted' and ever-changing.

In the purely statistical type of conception, the liquid is regarded as being essentially molecularly homogeneous. There is, however, a tendency for the molecules to concentrate in certain favourable positions with respect to neighbouring molecules. In other words, there is a maximum probability of nearby molecules being situate in certain more or less well-defined positions with respect to one another. The distribution function representing the statistical radial distribution of molecules around an average molecule possesses peaks which become less marked as the distance increases. Moreover, there appears in many cases to be a distinct resemblance between the form of this function and that of the equivalent discrete function by which the structure of the crystalline solid may be represented.

A valuable insight into the nature of liquid structure can be obtained from a consideration of modern theories of fusion. These deal with fusion as a type of order-disorder process, analogous to that which takes place in certain solid alloys, but with the difference that the lattice itself, as it were, melts. In more general terms, the process of fusion may be included with the other so-called 'cooperative' processes, the statistical mechanics of which have been studied by Guggenheim and Fowler. The obvious corollary to this conception is that a certain degree of regularity must persist in the liquid. Similar considerations applied to the reverse process of freezing should be capable of giving more fundamental information into the mechanism of supercooling and nucleus formation than is obtained from either the conceptions of Miers or of Tammann, and it is obvious that much valuable work remains to be done on this side of the problem.

The most direct evidence favouring the above views of liquid structure, although there is a body of more circumstantial evidence from other sources, is that obtained by X-ray studies. The halo or haloes produced when a monochromatic beam of $\mathrm{X}$-rays is passed through a liquid were early shown by Debye and Scherrer to be due to interference. Moreover, the Bragg spacing calculated from the most intense halo is in general of the same order as for the principal plane in the crystalline solid.

There is no space here to go fully into the question of the interpretation of the $\mathrm{X}$-ray diagrams, but two main methods may be very briefly described: (1) a 'model' of the liquid structure, based on the influence of the thermal vibrations on the structure of the crystal, may first be proposed, the theoretical $\mathrm{X}$-ray intensity diagram derived from this, and then compared with the actual diagram. (2) From what is essentially a Fourier analysis of the $\mathrm{X}$-ray intensity diagram the distribution function of the liquid is obtained. Naturally, further interpretation, and even further data, are necessary for this distribution function to be made to yield a detailed picture of the liquid structure. This latter is probably the most difficult aspect of the matter.

$\mathrm{X}$-ray studies have as yet been applied to only a few metals in the liquid state, but it seems already possible to draw certain tentative conclusions.

Of the close-packed metals lead, thallium and aluminium have been examined. All investigators agree that the liquids have a close-packed structure; that is to say, the atoms may be imagined as equal spheres which, while in continual motion, tend to remain always closely packed together. The inference is that there occur in the liquid, configurations bearing a relationship to those in the crystalline solid.

The body-centred cubic alkali metals have received considerable attention. Tarasov and Warren, for example, have shown that there exists a similarity between the atomic distribution of liquid and solid sodium. Their work has been followed up by Trimble and Gingrich, on the basis of whose results Wall has derived an interesting form of distribution function for liquid sodium. In his model each atom is imagined as being trapped in a spherical shell, the shells themselves being dis- 
tributed in an arrangement of a 'quasi-solid' type. He shows that the chief effect of temperature is in influencing the shell radius, this being $0.60 \mathrm{~A}$. at $100^{\circ} \mathrm{C}$. and $0.77 \mathrm{~A}$. at $400^{\circ} \mathrm{C}$. One may perhaps conclude from this that a fairly high degree of regularity, related to the body-centred cubic arrangement in the solid, may be present near the freezing-point.

Coming to metals deviating more markedly from close-packing in the solid state, mercury, naturally, has been studied by many investigators. All agree in imputing to liquid mercury a close-packed structure. Solid mercury is rhombohedral, with a coordination number of 6 , and the structure of the liquid may perhaps be most simply regarded as the result of the increased freedom given to the atoms on melting. Kratky has argued that a hexagonal close-packing fits the results best, but it seems unlikely that there can be much real difference between hexagonal and cubic close-packing in a structure so irregular as that of a liquid.

Bismuth is of particular interest in view of the large amount of work, in other connexions, of Goetz and his collaborators on this metal in the liquid state. There is some evidence of so-called 'premelting' in bismuth, and its melting point would appear to be not so sharp as for the close-packed metals. The results of the few X-ray investigations are conflicting. Randall and Rooksby find a band spacing identical with that for liquid lead, and consider that liquid bismuth has a close-packed structure. Solid bismuth has a structure differing widely from that of the close-packed metals, but, as Randall and Rooksby have pointed out, there are certain similarities to lead, its neighbour in the periodic system. Their view may be said to amount to the conclusion that this similarity increases on melting. They cite the changes in diamagnetism and electrical conductivity as affording more evidence in favour of this view, The results of Prins agree with the close-packed theory, but Danilov and Radtchenko consider that a 'blurred' simple cubic lattice fits their results best. The structure of solid bismuth is often considered as a slightly distorted simple cubic one.

As a result of his experiments, Goetz, particularly in connexion with his 'block' theory of the structure of crystals, has put forward the view that in liquid bismuth there persist, up to about $10^{\circ} \mathrm{C}$. above the melting-point, groups of atoms, under certain conditions quite large, having a highly regular arrangement closely allied to the structure of the solid. His theory has received considerable criticism, and it would seem that more certain evidence is necessary to substantiate it. To me, it seems a possibility that the change which takes place in bismuth on melting is, from one point of view, analogous to an order-disorder change in a solid alloy, accompanied (as is not infrequent) by a change in lattice form. The possibility of cybotaxis also exists.

X-ray investigations have also been made on liquid tin, but will not be dealt with here. Of perhaps greater interest are the results on gallium, as representing a 'borderline' case, or highly abnormal metal ; also because Menke has carried out an investigation on the supercooled liquid, at $18^{\circ} \mathrm{C} . \quad\left(\mathrm{m} . \mathrm{p} .=30^{\circ} \mathrm{C}\right.$. $) . \quad$ The intensity diagram shows a pronounced 'ridge' near the principal maximum. A similar effect occurs with water. The structure of solid gallium is complex, and there is evidence of molecular binding. The simplest conclusion is that some degree of molecular binding and relationship to the solid, possibly with cybotaxis, occurs in the liquid. On the other hand, it also seems not unlikely that a definite change in structural characteristics occurs on melting. It is noteworthy that supercooling would seem to be more marked in those metals, for example, bismuth and gallium, in which such a change takes place, than in metals such as the close-packed ones, in which the structural characteristics of liquid and solid appear to be more closely allied.

A few alloys have also been investigated in the liquid state by $\mathrm{X}$-ray methods. The results on intermetallic compounds indicate a certain degree of stability in the liquid state. Bornemann's measurements of the electrical conductivity of liquid alloys also confirm this. In view of the electron-atom ratio theory of the structure of intermetallic compounds, these results are of interest in indicating that the forces responsible for the structure of solid intermetallic compounds persist. in modified form in the liquid. It seems possible also that what amounts to a form of cybotaxis is present, the compounds, as it were, continually forming and dissociating. A few eutectic alloys have also been investigated. The results would seem to indicate the possibility that there is some tendency for the eutectic components to separate in the liquid, particularly near the freezing point ; that is, the structure of the solid alloy is to a certain extent 'foreshadowed' in the liquid. Again, what amounts to a form of cybotaxis, continual intermingling and separation of the eutectic components, seems possible.

It is evident that much work remains to be done. The experimental difficulties, quite apart from the difficulties of interpretation, are of course very great. Nevertheless, considering that the vast majority of metals and alloys used in industry start their existence, so to speak, in the liquid form, and that the process of freezing, as in casting, etc., is still so imperfectly understood, it would seem that there is here a useful line of fundamental research waiting to be developed. 